hep-th/0308055

SLAC-PUB-9669

SU-ITP-03/18

TIFR/TH/03-06

\title{
Towards Inflation in String Theory
}

\author{
Shamit Kachru, ${ }^{a, b}$ Renata Kallosh, ${ }^{a}$ Andrei Linde, ${ }^{a}$ \\ Juan Maldacena, ${ }^{c}$ Liam McAllister, ${ }^{a}$ and Sandip P. Trivedi ${ }^{d}$ \\ ${ }^{a}$ Department of Physics, Stanford University, Stanford, CA 94305, USA \\ ${ }^{b}$ SLAC, Stanford University, Stanford, CA 94309, USA \\ ${ }^{c}$ Institute for Advanced Study, Princeton, NJ 08540, USA \\ ${ }^{d}$ Tata Institute of Fundamental Research, Homi Bhabha Road, Mumbai 400 005, INDIA
}

We investigate the embedding of brane inflation into stable compactifications of string theory. At first sight a warped compactification geometry seems to produce a naturally flat inflaton potential, evading one well-known difficulty of brane-antibrane scenarios. Careful consideration of the closed string moduli reveals a further obstacle: superpotential stabilization of the compactification volume typically modifies the inflaton potential and renders it too steep for inflation. We discuss the non-generic conditions under which this problem does not arise. We conclude that brane inflation models can only work if restrictive assumptions about the method of volume stabilization, the warping of the internal space, and the source of inflationary energy are satisfied. We argue that this may not be a real problem, given the large range of available fluxes and background geometries in string theory.

skachru@stanford.edu, kallosh@stanford.edu, alinde@stanford.edu, malda@ias.edu, lpm@itp.stanford.edu, sandip@tifr.res.in 


\section{Introduction}

Inflation provides a compelling explanation for the homogeneity and isotropy of the universe and for the observed spectrum of density perturbations [1,2]. For this reason, we would hope for inflation to emerge naturally from any fundamental theory of microphysics. String theory is a promising candidate for a fundamental theory, but there are significant obstacles to deriving convincing models of inflation from string theory.

One problem is that string compactifications come with moduli fields which control the shape and size of the compactification manifold as well as the string coupling. Inflation is possible only if these fields are either stable or else have relatively flat potentials which do not cause fast, non-inflationary rolling in field space. Controlling the moduli in this way is a difficult problem. In particular, the potential for the dilaton and for the compactification volume tends to be a rather steep function [3].

A second problem is that the inflaton potential itself must be exceptionally flat to ensure prolonged slow-roll inflation. A successful microphysical theory would naturally produce such a flat potential. Since the flatness condition for the potential involves the Planck scale one should ensure that quantum gravity corrections do not spoil it. Hence, the problem should be analyzed in a theory of quantum gravity, such as string theory. The hope of brane-antibrane inflation scenarios is that the brane-antibrane interaction potential can play the role of the inflaton potential (see [⿴囗十 for a nice review), but it is well known that this potential is not naturally flat. Since in string theory one cannot finetune by hand, but only by varying background data (like the compactification manifold or the choice of flux), one concludes that in generic compactifications, brane inflation will not work. However, the many choices of flux and compactification make possible a considerable degree of discrete fine-tuning, so for very special choices of the background one would expect to find potentials which are sufficiently flat for inflation.

In this note we discuss these problems in the concrete context of the warped type IIB compactifications described in e.g. [5, 60. One reason for working in this setting is that one can sometimes stabilize all the moduli in a geometry of this type, avoiding the first problem mentioned above. In addition, the constructions of [5] naturally admit D3-branes and anti-D3-branes transverse to the six compact dimensions. Furthermore, one could wish for a model which accommodates both inflation and the present-day cosmic acceleration. This might be possible if one could construct inflationary models which asymptote at late 
times to the de Sitter vacua of [7] (or variants on that construction, as described in e.g. [8,9]; earlier constructions in non-critical string theory appeared in [10]). As these vacua included one or more anti-D3-branes in a warped type IIB background, it is quite natural to consider brane-antibrane inflation in this context.

Our idea, then, is to begin with the de Sitter vacua constructed in [7], add a mobile D3-brane, and determine whether the resulting potential is suitable for inflation. For the impatient reader, we summarize our findings here. We find that modest warping of the compactification geometry produces an extremely flat brane-antibrane interaction potential, provided that we neglect moduli stabilization. This solves the second problem listed above. However, a new problem appears when we incorporate those terms in the potential which led, in the construction of [7], to the stabilization of the volume modulus. We show that generic volume-stabilizing superpotentials also impart an unacceptably large mass to the inflaton, halting inflation.

While these conclusions are "generic," it is very important to emphasize that the problem of the inflaton mass might be circumvented in at least two different ways. First, the stabilization mechanism for the moduli might be different from that in [7]. For example, the volume modulus could be stabilized by corrections to the Kähler potential, which, as we will see, can naturally circumvent this problem. Second, the mobile brane might be located not at a generic point in the compact manifold but close to some preferred point. If the location of the D3-brane is appropriately chosen then there could be significant corrections to the superpotential. In general models, the superpotential may be a rather complicated function of both the brane positions and the volume modulus. Little is known about the form of these nonperturbative superpotentials in string compactifications. Our arguments show that if the functional form of the superpotential is generic then inflation does not occur. Nevertheless, it seems quite likely, given the range of available fluxes and background geometries, that cases exist which are sufficiently non-generic to permit inflation, although with predictions which are altered from those of naive brane inflation. 1

Our conclusions should be viewed as a first pass through the class of brane inflation models, in the context of the moduli stabilization mechanism which has recently been

1 This point is made more quantitative in Appendix F, where we explain that the degree of non-genericity required corresponds roughly to a fine-tune of one part in 100. 
developed in [5,7]. Once the non-perturbative superpotentials involved in such constructions are better understood, and/or as soon as other mechanisms for moduli stabilization become available, one could re-examine brane inflation in light of this further concrete knowledge. This may well lead to a precise determination of the non-generic cases where working models of brane inflation in string theory can be realized.

Our analysis clearly indicates that any viable inflation scenario in string theory has to address the moduli stabilization problem. Since essentially all papers on the subject, to the best of our knowledge, have ignored the problem of moduli stabilization, their conclusions are questionable in view of our results. In particular, should a more detailed analysis reveal the possibility of inflation in various non-generic situations, as suggested above, we expect that the resulting inflationary parameters will typically be quite different from those calculated in the existing literature by neglecting moduli stabilization.

This paper is organized as follows. In $\S 2$ we review basic facts about brane-antibrane inflation [11,12], with special attention to the case of D3-branes, and discuss some generic problems for such models. In $\S 3$ we show that warping of the geometry can help with some of these problems. In $\S 4$ we explain one method of embedding the warped inflation scenario into string theory, using the warped compactifications of [5]. In $\$ 5$ we describe further problems that arise in the string theory constructions when one tries to stabilize the overall volume modulus. Generic methods of stabilization (e.g. via a nonperturbative superpotential) modify the inflaton potential and make inflation difficult to achieve. We discuss several ways to overcome this problem. We conclude with some general remarks in $\S 6$.

Appendix A contains a general discussion of the gravitational interaction of an (unwarped) brane-antibrane pair, and demonstrates that the potentials which arise are typically not flat enough to lead to prolonged inflation. In Appendix B we specialize to a warped background and derive the interaction potential. In Appendix $\mathrm{C}$ we explore the detailed properties of inflation in warped brane-antibrane models, assuming that a solution to the challenges of $\S 5$ has been found. In Appendix D we explain that eternal inflation may be possible in this scenario. In Appendix E we discuss the exit from inflation and point out that the production of undesirable metric perturbations due to cosmic strings, which are typically created during brane-antibrane annihilation, is highly suppressed in warped models. Finally, in Appendix F we discuss the possibility of fine-tuning of the inflaton potential in order to achieve an inflationary regime. 
After completing this work, we became aware of the papers [13], in which related issues are addressed.

\section{Brief Review of $D 3 / \overline{D 3}$ Inflation}

In brane-antibrane inflation one studies the relative motion of a brane and an antibrane which are initially separated by a distance $r$ on the compactification manifold $M$. One should assume $r \gg l_{s}$, so that the force is well approximated by the Coulomb attraction due to gravity and RR fields. Then the potential takes the form

$$
V(r)=2 T_{3}\left(1-\frac{1}{2 \pi^{3}} \frac{T_{3}}{M_{10, P l}^{8} r^{4}}\right) .
$$

where $M_{10, P l}$ is the ten-dimensional Planck scale, defined by $8 \pi G_{10, N}=M_{10, P l}^{-8}$, and $T_{3}$ is the tension of a D3-brane. In terms of a canonically normalized scalar field $\phi$, one can rewrite this as

$$
V(\phi)=2 T_{3}\left(1-\frac{1}{2 \pi^{3}} \frac{T_{3}^{3}}{M_{10, P l}^{8} \phi^{4}}\right) .
$$

It was suggested in [11] that for large fields (large $r$ ), one may obtain inflation from this potential.

A basic (and well known [4] problem with this scenario is the following. The standard inflationary slow-roll parameters $\epsilon$ and $\eta$ are defined via

$$
\begin{gathered}
\epsilon \equiv \frac{M_{P l}^{2}}{2}\left(\frac{V^{\prime}}{V}\right)^{2} \\
\eta \equiv M_{P l}^{2} \frac{V^{\prime \prime}}{V} .
\end{gathered}
$$

One generally wants $\epsilon, \eta \ll 1$ to get slow-roll inflation with sufficient e-foldings. Is this possible in the model (2.2)? The four-dimensional Planck mass appearing in (2.4) is $M_{P l}^{2}=M_{10, P l}^{8} L^{6}$ where $L^{6}$ is the volume of M. This implies that $\eta$ is

$$
\eta=-\frac{10}{\pi^{3}}(L / r)^{6} \sim-0.3(L / r)^{6}
$$

Hence, $\eta \ll 1$ is possible only for $r>L$ - but two branes cannot be separated by a distance greater than $L$ in a manifold $M$ of size $L$ ! 
One can try to evade this constraint by considering anisotropic extra dimensions or non-generic initial conditions which yield flatter potentials than (2.2). We argue in Appendix A that this is not possible. There are always some tachyonic directions in the potential with $\eta \leq-2 / 3$. This implies that the slow-roll approximation cannot be maintained for a large number of e-foldings.

In $\S 3$ we will explore another possibility that successfully evades this problem - we will modify the potential (2.2) by considering branes and antibranes in a warped geometry. We should mention that there are other proposals which might evade the above problem, such as branes at angles or branes with fluxes, see [14, 15, 16, 17, 18.

However, all of these models have an unsolved problem: moduli stabilization. For an internal manifold of size $L$, the correct four-dimensional Einstein-frame potential is not quite (2.2). If one assumes that the main contribution to the inflationary energy comes from the D3-brane tension then one finds, for $r \gg l_{s}$, that

$$
V(\phi, L) \sim \frac{2 T_{3}}{L^{12}}
$$

The energy in the brane tensions sources a steep potential for the radial modulus $L$ of the internal manifold. Therefore, in the absence of a stabilization mechanism which fixes $L$ with sufficient mass so that the variation of $L$ in (2.6) is negligible, one will find fast-roll in the direction of large $L$ rather than slow-roll in the direction of decreasing $r$. This means that it is important to study concrete scenarios where the volume modulus has already been stabilized. However, we will show that not every means of volume stabilization is compatible with inflation, even when the naive inter-brane potential is flat enough to inflate. We will return to the issue of volume stabilization in $\S 5$, where we will discuss a new and generic problem which appears when one considers the issue in detail.

\section{Inflation in a Warped Background: Essential Features}

Our modified brane-antibrane proposal is that inflation might arise from the interaction potential between a D3-brane and an anti-D3-brane which are parallel and widely separated in five-dimensional anti de Sitter space $\left(\mathrm{AdS}_{5}\right)$.2

2 This is a slight simplification; in $\S 4$ we will construct compact models which deviate from $\mathrm{AdS}_{5}$ both in the infrared and in the ultraviolet. It is nevertheless convenient to work out the essential features of the model in this simpler geometry. 
The anti-D3-brane is held fixed at one location in the infrared end of the geometry (this is naturally enforced by the dynamics, as we shall explain). The D3-brane is mobile; it experiences a small attractive force towards the anti-D3-brane. The distance between the branes plays the role of the inflaton field.

The forces on the brane and antibrane arise as follows. A single D3-brane experiences no force in an AdS background: electrostatic repulsion from the five-form background exactly cancels gravitational attraction. The addition of a distant anti-D3-brane results in a relatively weak interaction potential arising from the attraction between the brane and the antibrane. We interpret this as a slowly varying potential for the inflaton. We will demonstrate in $\S 3.2$ and in Appendix B that this potential is much flatter than the interaction potential for a brane-antibrane pair in flat space.

In the remainder of this section we explain this key idea in more detail. $\S 3.1$ is a review of gravity in a warped background. $\S 3.2$ deals with the motion of a brane probe in such a background.

It is important to point out that throughout this discussion, we will ignore the possibility that other moduli (or the effects which stabilize them) interfere with inflation. In the context of the string constructions of $\S 4$, the relevant other modulus is the compactification volume, and the generic problems associated with its stabilization are the subject of $\S 5$. In fact we will see that this modulus problem will generically stop inflation.

\subsection{Gravity in an AdS Background}

We first consider a compactification of string theory on $\mathrm{AdS}_{5} \times \mathrm{X}_{5}$ where $\mathrm{X}_{5}$ is a five-dimensional Einstein manifold. 3 This arises in string theory as a solution of tendimensional supergravity coupled to the five-form field strength $F_{5}$. The $\mathrm{AdS}_{5}$ solution is given in Poincaré coordinates by the metric

$$
d s^{2}=\frac{r^{2}}{R^{2}}\left(-d t^{2}+d \vec{x}^{2}\right)+\frac{R^{2}}{r^{2}} d r^{2}
$$

There is, in addition, a five-form flux: if the geometry (3.1) arises as the near-horizon limit of a stack of $N$ D3-branes, then the five-form charge (in units of the charge of a single

3 The detailed form of $\mathrm{X}_{5}$ will not matter for the moment. For concreteness the reader may imagine that $\mathrm{X}_{5}=\mathrm{S}^{5}$. 
D3-brane) is $N . R$, the characteristic length scale of the $\mathrm{AdS}_{5}$ geometry, is related to the five-form charge by

$$
R^{4}=4 \pi a g_{s} N \alpha^{\prime 2}
$$

where the constant $a$ depends on $X_{5}$. It will be useful to recall that AdS is a maximally symmetric, constant curvature spacetime. Its curvature scales like $\frac{1}{R^{2}}$ and is independent of the radial location $r$. As long as $N \gg 1$ this curvature is small and supergravity analysis is reliable. We will choose to truncate $\mathrm{AdS}_{5}$ to the region $r_{0}<r<r_{\max }$.

The reader will notice that, apart from the additional manifold $\mathrm{X}_{5}$, this background is identical to that considered by Randall and Sundrum in [19]. Two physical insights from [19] will be crucial for our model. First, one can see from the warped metric (3.1) that the region of small $r$ is the bottom of a gravitational well. Energies along the $t, x^{i}$ coordinates therefore get increasingly redshifted as $r$ decreases. (The region of significant redshift is consequently referred to as the infrared end of the geometry.) Second, as a result of truncating the AdS region, the four-dimensional effective theory which governs low-energy dynamics will have a finite gravitational constant, and will include four-dimensional gravity described by the Einstein-Hilbert action: $⿴$

$$
S_{\text {grav }}=\frac{1}{16 \pi G_{N}} \int d^{4} x \sqrt{-g} \mathcal{R}
$$

Recall also that in [19], the truncation of AdS space was achieved in a brute force manner by placing two branes, conventionally called the Planck brane and the Standard Model brane, at $r_{\max }$ and $r_{0}$, respectively. In the string theory constructions of [5], the truncation arises because the compactification geometry departs significantly from that of $\mathrm{AdS}_{5} \times \mathrm{X}_{5}$ away from the region $r_{0}<r<r_{\max }$. In the ultraviolet, in the vicinity of $r \geq r_{\max }$, the AdS geometry smoothly glues into a warped Calabi-Yau compactification. In the infrared, near $r=r_{0}$, the AdS region often terminates smoothly (as in the example of [20]). The infrared smoothing prevents the redshift factor $r / R$ from decreasing beyond a certain minimum whose value will be very important for our model.

4 The graviton zero modes have polarizations parallel to $t, x_{i}$, are constant on $\mathrm{X}_{5}$, and have a profile identical to the warped background. 


\subsection{Brane Dynamics}

We mentioned above that the warped nature of the geometry gives rise to a redshift dependent on the radial location. It will be important in the discussion below that the redshift results in a very significant suppression of energies at the location of the antibrane; that is, the ratio $r_{0} / R$ is very small. Also note that within the truncated AdS geometry, $r_{0}<r<r_{\max }$, we have chosen to place the anti-D3-brane at the infrared cutoff $r=r_{0}$, where it has minimum energy due to the redshift effect.

The five-form background is given by

$$
\left(F_{5}\right)_{r t x^{1} x^{2} x^{3}}=\frac{4 r^{3}}{R^{4}}
$$

In a suitable gauge the corresponding four-form gauge potential $C_{4}$ takes the form

$$
\left(C_{4}\right)_{t x^{1} x^{2} x^{3}}=\frac{r^{4}}{R^{4}}
$$

The D3-brane stretches along the directions $t, x^{1}, x^{2}, x^{3}$. Its location in the radial direction of AdS space will be denoted by $r_{1}$. In the discussion below we will assume (self-consistently) that the D3-brane has a fixed location along the angular coordinates of the $\mathrm{X}_{5}$ space. The motion of the D3-brane is then described by the Born-Infeld plus Chern-Simons action

$$
S=-T_{3} \int \sqrt{-g} d^{4} x\left(\frac{r_{1}^{4}}{R^{4}}\right) \sqrt{1-\frac{R^{4}}{r_{1}^{4}} g^{\mu \nu} \partial_{\mu} r_{1} \partial_{\nu} r_{1}}+T_{3} \int\left(C_{4}\right)_{t x^{1} x^{2} x^{3}} d t d x^{1} d x^{2} d x^{3}
$$

The indices $\mu, \nu$ denote directions parallel to the D3-brane along the $t, x^{1}, x^{2}, x^{3}$ coordinates, and $g^{\mu \nu}$ is the metric along these directions. The D3-brane tension, $T_{3}$, is

$$
T_{3}=\frac{1}{(2 \pi)^{3} g_{s} \alpha^{2}}
$$

For future purposes we note here that since an anti-D3-brane has the same tension as a D3-brane but opposite five-form charge, it is described by a similar action where the sign of the second term is reversed.

Now consider a D3-brane slowly moving in the background given by (3.1) and (3.4), with no antibranes present. It is easy to see that because of a cancellation between the 
Born-Infeld and Chern-Simons terms, the D3-brane action at low energies is just that of a free field,

$$
S=T_{3} \int d^{4} x \sqrt{-g} \frac{1}{2} g^{\mu \nu} \partial_{\mu} r_{1} \partial_{\nu} r_{1} .
$$

This in accord with our comment above that the net force for a D3-brane in the background (3.1),(3.4) vanishes due to gravitational and five-form cancellations.

We are now ready to consider the effect of an antibrane on the D3-brane. Physically this arises as follows. The anti-D3-brane has a tension and a five-form charge and perturbs both the metric and the five-form field. This in turn results in a potential energy dependent on the location of the D3-brane.

The potential between a brane located at $r_{1}$ and an antibrane located at $r_{0}$, in the limit when $r_{1} \gg r_{0}$, is given by:

$$
V=2 T_{3} \frac{r_{0}^{4}}{R^{4}}\left(1-\frac{1}{N} \frac{r_{0}^{4}}{r_{1}^{4}}\right) .
$$

For a derivation see Appendix B.

The first term in the potential is independent of the location of the D3-brane and can be thought of as a constant potential energy associated with the anti-D3-brane. It is proportional to the tension $T_{3}$. For the antibrane the force exerted by gravity and the fiveform field are of the same sign and add, so we have a factor of 2 . In addition, the warped geometry gives rise to a redshift, which reduces the effective tension of the antibrane by a factor $r_{0}^{4} / R^{4}$.

The second term in (3.9) depends on the location of the D3-brane; its negative sign indicates mutual attraction between the pair. Two features of this term will be important in the subsequent discussion. First, the term varies slowly, as the inverse fourth power of the radial location of the D3-brane. Second, due to the warping of the background, the coefficient of this second term is highly suppressed, by a redshift factor $r_{0}^{8} / R^{4}$.

Two more comments are in order at this stage. We have assumed that the antibrane is fixed at $r_{0}$. From (3.9), we see that this is in fact a good approximation to make. In the $r_{1} \gg r_{0}$ limit the first term in (3.9) is much bigger than the second, and most of the energy of the anti-D3-brane arises due to interaction with the background. This is minimized when the anti-D3-brane is located at $r_{0}$ in the truncated AdS spacetime. Second, in our analysis above, we are working in the approximation $r_{1} \gg r_{0}$. We will 
see below that the D3-brane is far away from the anti-D3-brane while the approximately sixty e-foldings of inflation occur, so this condition is met during the inflationary epoch. Eventually the D3-brane approaches the antibrane, $r_{1} \sim r_{0}$, and this approximation breaks down. The potential then becomes quite complicated and more model dependent (e.g. it depends on the separation between the brane and antibrane along $\mathrm{X}_{5}$ ). The resulting dynamics is important for reheating.

A summary of the discussion so far is as follows. We have considered a D3-brane moving in an $\mathrm{AdS}_{5} \times \mathrm{X}_{5}$ background with five-form flux, in the presence of a fixed antiD3-brane. This system is described by an action:

$$
S=\int d^{4} x\left(\frac{1}{2} T_{3} g^{\mu \nu} \partial_{\mu} r_{1} \partial_{\nu} r_{1}-2 T_{3} \frac{r_{0}^{4}}{R^{4}}\left(1-\frac{1}{N} \frac{r_{0}^{4}}{r_{1}^{4}}\right)\right)
$$

The reader will notice in particular that $r_{1}$, the location of the D3-brane, is a scalar field in the effective four-dimensional theory.

Once we cut off the $\mathrm{AdS}_{5}$ space as in the Randall-Sundrum models we will find that we can add to (3.10) the four-dimensional Einstein action. However, we should also add an extra coupling of the form $\frac{T_{3}}{12} r_{1}^{2} \mathcal{R}$ coming from the fact that the scalar field $r_{1}$ describing the position of the D3-brane is a conformally coupled scalar [21]. This unfortunately leads to a large contribution to $\eta$. We will discuss this phenomenon in more generality (from the perspective of the effective low-energy four-dimensional supergravity) in $\S 5$.

The model described above has several appealing features in addition to the flatness of the potential. We study these properties in Appendices C,D, and E, with the assumption that one can somehow overcome the problems of $\S 5$ (which must be tantamount to cancelling the conformal coupling). In Appendix $\mathrm{C}$ we compute the inflationary parameters and show that observational constraints are easily met. In Appendix D we argue that eternal inflation can be embedded into this model, and in Appendix E we point out that the warped geometry suppresses the production of metric perturbations due to cosmic strings (which naturally form during the brane/anti-brane annihilation).

\section{A Concrete Example in String Theory}

We now show how to realize our proposal in a specific class of string compactifications. In $\S 4.1$ we present the compactifications and explain why they contain warped throat 
regions. As the warped throat is well-described by the Klebanov-Strassler (KS) solution [20], we dedicate $\S 4.2$ to a very brief review of the KS geometry. In $\S 4.3$ we show that a brane moving in the $\mathrm{KS}$ background might give rise to inflation, realizing the general idea presented in $\S 3$. Throughout this discussion, we ignore the problem of stabilizing the overall volume modulus, which is unfixed in the constructions of [5]. We consider the problem of volume stabilization in $\S 5$, where we will find that generic methods of volume stabilization can perturb the inflaton enough to stop inflation.

\subsection{The Compactification}

Our starting point is type IIB string theory compactified on a six-dimensional CalabiYau orientifold. More generally one could use F-theory on an elliptically-fibered Calabi-Yau fourfold. We choose to turn on background fluxes: the three-form fluxes $F_{3}, H_{3}$ present in the theory are placed along cycles in the internal space (and $F_{5}$ is fixed as in [5]). These fluxes induce warping of the background. One can show that the resulting space is a warped product of Minkowski space and the Calabi-Yau:

$$
d s^{2}=e^{2 A(y)} \eta_{\mu \nu} d x^{\mu} d x^{\nu}+e^{-2 A(y)} g_{m n} d y^{m} d y^{n}
$$

where $y_{i}$ are coordinates on the compactification manifold and $g_{m n}$ is the Calabi-Yau metric. As was discussed in [5], one expects that with a generic choice of flux, all the complex structure moduli of the Calabi-Yau, as well as the dilaton-axion, will be fixed. We will assume that the compactification has only one Kähler modulus, the overall volume of the internal space.

As described in [5], one can use the above construction to compactify the warped deformed conifold solution of Klebanov and Strassler (KS). We spend the next section reviewing a few facts about this geometry, as certain details will be important for inflation.

\subsection{The Klebanov-Strassler Geometry}

The Klebanov-Strassler geometry [20] is a noncompact ten-dimensional solution to type IIB supergravity in the presence of background fluxes. The spacetime naturally decomposes into a warped product of a Minkowski factor and a six-dimensional internal space. The six-dimensional space has a tip which is smoothed into an $S^{3}$ of finite size. Far 
from this tip the geometry can be approximated by a cone over the Einstein manifold $T^{1,1}$, which is topologically $S^{2} \times S^{3}$. Our coordinates will be five angles on $T^{1,1}$, which we can consistently neglect in the following, and a radial coordinate $r$ which measures distance from the tip. The background fluxes are given by

$$
\frac{1}{(2 \pi)^{2} \alpha^{\prime}} \int_{A} F=M, \quad \frac{1}{(2 \pi)^{2} \alpha^{\prime}} \int_{B} H=-K
$$

where A is the $S^{3}$ at the tip and B is its Poincaré-dual three-cycle. We will require that $M \gg 1$ and $K \gg 1$; these conditions are important in deriving the solution. The exact metric is known, but for our purposes a simpler form, valid far from the tip, will be more useful. For large $r$ we may express the complete ten-dimensional solution as

$$
d s^{2}=h^{-1 / 2} \eta_{\mu \nu} d x^{\mu} d x^{\nu}+h^{1 / 2}\left(d r^{2}+r^{2} d s_{T^{1,1}}^{2}\right)
$$

where now

$$
h(r)=\frac{27 \pi}{4 r^{4}} \alpha^{\prime 2} g_{s} M\left(K+g_{s} M\left(\frac{3}{8 \pi}+\frac{3}{2 \pi} \ln \left(\frac{\mathrm{r}}{\mathrm{r}_{\max }}\right)\right)\right) .
$$

Neglecting the logarithmic corrections and the second term on the right, this takes the form 5

$$
\begin{gathered}
R^{4}=\frac{27}{4} \pi g_{s} N \alpha^{\prime 2} \\
N \equiv M K
\end{gathered}
$$

When the KS geometry is embedded in a compactification then at some location $r=r_{\max }$ the warped throat geometry is smoothly joined to the remainder of the warped CalabiYau orientifold. Near this gluing region, departures from the $\operatorname{AdS}_{5} \times \mathrm{T}^{1,1}$ geometry are noticeable; eventually the AdS must end. In terms of redshift this location corresponds to the deep ultraviolet, and so the gluing region plays the role of the ultraviolet cutoff (Planck brane) in the AdS of $\S 3$.

The exact solution likewise shows departures from (4.3) in the far infrared, near the

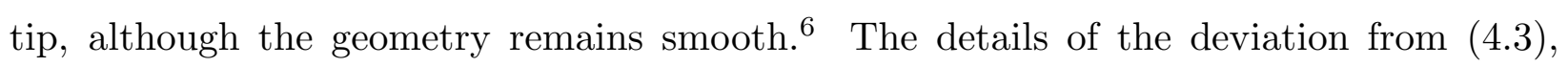
although known, are unimportant here; it will suffice to know the redshift at the tip. This can be modeled by cutting off the radial coordinate at some minimum value $r_{0}$, which is the location of the tip. It was shown in [5] that the minimal redshift satisfies

$$
\frac{r_{0}}{R}=e^{-\frac{2 \pi K}{3 g_{s} M}}
$$

This can be extremely small given a suitable choice of fluxes.

5 The second term on the r.h.s. of (4.4) can easily be included. For the numerical values discussed in Appendix $\mathrm{C}$, this gives a three percent correction.

6 The radius of curvature is $\sqrt{g_{s} M \alpha^{\prime}}$, so the tip is smooth provided $g_{s} M \gg 1$. 


\subsection{Inflation from Motion in the KS Region}

In [7] additional anti-D3-branes were introduced in the KS region. These anti-D3branes minimize their energy by sitting at the location where the redshift suppression is maximum, i.e. at the very tip of the deformed conifold, where $r \sim r_{0}$ (the dynamics of anti-D3-branes in the KS geometry was studied in [22]).

Thus we see that the string construction outlined above has all the features of the general model of $\S 3$ : a truncated $\mathrm{AdS}_{5}$ geometry, an associated five-form flux of the correct strength, and anti-D3-branes fixed at the location of maximum redshift. In addition most of the moduli associated with the compactification, including the dilaton, are stabilized. The one exception is the volume modulus; we will discuss the complications its stabilization introduces separately, in $\S 5$.

No mobile D3-branes were included in the construction of [7], but it is easy to incorporate them. One needs to turn on somewhat different values of three-form flux, which allow the four-form tadpole to cancel in the presence of the additional D3-branes. This is straightforward to do and does not change any of the features discussed above.

We will take one such D3-brane to be present in the KS region of the compactification. The general discussion of $\S 3$ applies to this brane. Since the D3-brane is described by the action (3.6), with $R$ now given by (4.5), the calculation of the brane-antibrane potential follows the discussion in Appendix C, which we outline here.

In the KS model the warp factor (4.1) is given in terms of a function $h \equiv e^{-4 A}$ which obeys a Laplace equation, with the fluxes and branes acting as sources. In particular, a single D3-brane located at $r=r_{1}$ will correct the background according to

$$
h_{\text {new }}(r)=h(r)+\Delta h\left(r, r_{1}\right) .
$$

Here $h(r)$ is the background given in (4.4) and $\Delta h\left(r, r_{1}\right)$ is the correction due to the D3brane. In a region where the original warp factor is very small we see that $h\left(r_{0}\right) \gg 1$, so that the total warp factor can be expanded as

$$
e^{4 A} \sim h\left(r_{0}\right)^{-1}\left(1-\frac{\Delta h\left(r, r_{1}\right)}{h\left(r_{0}\right)}\right) .
$$

This warp factor yields the contribution to the energy due to the presence of an antibrane. If $h\left(r_{0}\right) \gg 1$ this typically gives a very flat potential. 
The small warp factor and the consequent exponential flatness are the heart of our proposal, so an alternative explanation of the origin of these small numbers may be helpful. Recall that there is a holographic dual gauge theory which describes the geometry of the KS model. This gauge theory is approximately scale invariant in the deep ultraviolet, with slowly running gauge couplings. It undergoes $K$ duality cascades before leading in the infrared to a confining gauge theory with a mass gap. Then the smallness of the redshift factor,

$$
\left(\frac{r_{0}}{R}\right)^{4}=e^{-\frac{8 \pi K}{3 g_{s} M}}
$$

can be ascribed to the exponential smallness of the confinement scale in such a gauge theory.

In summary, we have seen that one can construct concrete examples of string compactifications which lead to the general behavior described in $\S 3$. One of their virtues is that they automatically lead to very flat inflaton potentials, without the need for large brane separation or excessive fine-tuning of initial conditions. The primary source of this flatness is the redshift suppression (4.7) which is exponentially sensitive to the (integer) choice of fluxes $K$ and $M$. However, all of these virtues must be re-examined in the light of concrete ideas about how to stabilize the closed string moduli. In this general class of flux compactifications, the fluxes stabilize many moduli but not e.g. the overall volume. We now turn to the discussion of volume stabilization.

\section{Volume Stabilization: New Difficulties for D-brane Inflation}

The results of $\S 3,4$ indicate that warped geometries provide a promising setting for making models of inflation with naturally small $\epsilon$ and $\eta$. However, as emphasized in $\S 2$, one must ensure that the compactification volume is stabilized in order to avoid rapid decompactification instead of inflation. We will now demonstrate that in the concrete models of [5] this is far from a trivial constraint.

In these models the four-dimensional $\mathcal{N}=1$ supergravity at low energies is of the no-scale type. The Kähler potential for the volume modulus $\rho$ and the D-brane fields $\phi$ takes the form $[6]^{\natural}$

$$
K(\rho, \bar{\rho}, \phi, \bar{\phi})=-3 \log (\rho+\bar{\rho}-k(\phi, \bar{\phi}))
$$

7 The variable $\rho$ is called $-i \rho$ in [7]. 
Let us pause for a moment to explain how this is obtained. In the tree level compactification the massless fields are the volume, the axion and the position $\phi$ of the branes. The axion comes from a four-form potential proportional to a harmonic four-form in the internal manifold [5]. At first sight one would think that the moduli space is simply a product of the moduli space for $\phi$, which is just the internal Calabi-Yau manifold, and the space spanned by the volume and the axion. This is not correct; the axion describes a circle which is non-trivially fibered over the $\phi$ moduli space. This structure arises from the coupling of the four-form potential to the worldvolume of the moving D3-brane. The moduli space has a metric of the form

$$
d s^{2}=\frac{3}{2 r^{2}}\left(d r^{2}+\left(d \chi+\frac{1}{2} i k_{, j} d \phi^{j}-\frac{1}{2} i k_{, j} d \bar{\phi}^{j}\right)^{2}\right)+\frac{3}{r} k_{, i \bar{j}} d \phi^{i} d \phi^{\bar{j}}
$$

where $r$ is proportional to the volume of the Calabi Yau (in the notation of [5], $r \sim e^{4 u}$ ). If we tried to work with a complex variable $r+i \chi$ then (5.2) would not follow from a Kähler potential. It turns out that the good complex variable is $\rho$, which is defined as follows. The imaginary part of $\rho$ is the axion, while the real part of $\rho$ is defined by

$$
2 r=\rho+\bar{\rho}-k(\phi, \bar{\phi})
$$

It is then possible to see that (5.1) gives rise to (5.2). This type of definition of $\rho$ arises when we Kaluza-Klein compactify supergravity theories; see for example [23].

The superpotential is of the form

$$
W=W_{0}
$$

where $W_{0}$ is a constant (we assume the D-branes are on their moduli space, so we do not write down the standard commutator term). This arises from the $(0,3)$ part of the three-form flux in the full theory including the complex structure moduli and the dilaton. We have not yet included the anti-D3-branes used in $\S 3,4$; these will be incorporated at the end of the discussion.

It is important that with the Kähler potential (5.1), one obtains the no-scale cancellation in the potential

$$
V=e^{K}\left(g^{a \bar{b}} K_{, a} K_{, \bar{b}}|W|^{2}-3|W|^{2}\right)=0
$$


since

$$
g^{a \bar{b}} \partial_{a} K \partial_{\bar{b}} K=3
$$

where $a, b$ run over $\rho$ and $\phi .8$

Using (5.5), it is clear that a generic $W(\phi)$ will yield a potential for the D-brane fields $\phi$, but that the potential for the $\rho$ modulus will vanish if the solution for the $\phi$ fields has $\partial_{\phi} W=0$. It is also clear that a constant superpotential, as in (5.4), gives no potential to the $\phi$ fields. This is consistent with the analysis in [5], where the pseudo-BPS nature of the flux background leaves the D3-brane moduli unfixed.

We are interested in finding a situation where the D-branes can move freely in the Calabi-Yau (so the $\phi$ fields are unfixed), but the volume is stabilized. Before we discuss various scenarios for such a stabilization, it is important to distinguish carefully between the $\rho$ chiral superfield, and the actual volume modulus, $r$, which controls the $\alpha^{\prime}$ expansion.

The Kähler potential (5.1) has the following peculiar feature. Let us imagine that there is one D-brane, and hence a triplet of fields $\phi$ describing its position on the CalabiYau space. Then $k(\phi, \bar{\phi})$ should be the Kähler potential for the Calabi-Yau metric itself, at least at large volume. However, under Kähler transformations of $k$, the expression (5.1) is not well behaved. This can be fixed by assigning the transformation laws

$$
k(\phi, \bar{\phi}) \rightarrow k+f(\phi)+\overline{f(\phi)}, \quad \rho \rightarrow \rho+f, \quad \bar{\rho} \rightarrow \bar{\rho}+\bar{f}
$$

This is a manifestation of the fact that the circle described by the axion is non-trivially fibered over the $\phi$ moduli space. Note that the physical volume of the internal dimensions, which is given by $r,(5.3)$, is invariant under (5.7).

Armed with this knowledge, and given (5.1) and (5.4) as our starting point, we can now explore various scenarios for volume stabilization.

8 The easiest way to check (5.6) is to note that in expression (5.6) we can switch back to the variables $r, a, \phi$ in (5.2). In these variables $K$ is only a function of $r$. 


\subsection{Scenario I: Superpotential Stabilization}

Perhaps the most straightforward method of stabilizing the volume involves a nonperturbative contribution to the superpotential. Various sources of nonperturbative superpotentials for the $\rho$ modulus are known; one instructive example described in [7] involves a superpotential

$$
W(\rho)=W_{0}+A e^{-a \rho}
$$

where $A$ and $a$ are constants and $W_{0}$ is the contribution (5.4) of the three-form flux. For the remainder of this section we will consider $W=W(\rho)$ to be a general holomorphic function of $\rho$.

In the presence of D3-branes the superpotential must in addition develop some dependence on $\phi$, as it should be invariant under (5.7). For instance, as argued in [24], the superpotential due to Euclidean brane instantons or gauge dynamics on D7-branes has to vanish when a D3-brane hits the relevant cycle. This can be understood directly by examining and integrating out the massive D3-D7 strings in the latter case. This subtlety must be accounted for to get a globally well-defined $W$, and we will see in a moment that this actually changes the inflaton mass term. Nevertheless, we will first study the simpler case $W=W(\rho)$, both because it reflects the essential features of the problem and because the full dependence of $W$ on $\phi$ is not known.

Let us start by presenting a general argument which highlights a problem faced by any inflationary model involving a moving D3-brane in the models of [7]. The main point is that one will choose some configuration with a positive energy $V$. When the compact manifold is large then this energy will go to zero rather quickly, as a power of the volume modulus $r$ :

$$
V(r, \phi)=\frac{X(\rho)}{r^{\alpha}}=\frac{X(\rho)}{(\rho-\phi \bar{\phi} / 2)^{\alpha}}
$$

where $\alpha$ is a number of order one and the form of $X(\rho)$ depends on the source of energy. This follows because in existing proposals the inflationary energy arises either from brane tensions or from fluxes, and all known brane and flux energies vanish as some power of $r$. On the other hand the stabilization mechanism would fix $\rho$ (or else some combination of $\rho$ and $\phi$ ) rather than $r$. This implies that as the brane moves and $\phi$ changes there will be a change in the potential,

$$
V=V_{0}\left(1+\alpha \frac{\phi \bar{\phi}}{2 r}+\ldots\right) .
$$


This will lead to a contribution to $\eta$ of order one, unless there is a compensating contribution to the mass term from some other source.

One possible source of such a cancellation is a dependence of the superpotential on $\phi$, not just $\rho$. If $V(r, \phi)=X(\rho, \phi) r^{-\alpha}$ then we would get an additional contribution to the mass term,

$$
V(\rho, \phi)=\frac{X(\rho, \phi)}{(\rho-\phi \bar{\phi} / 2)^{\alpha}}=\frac{X(\rho)}{\rho^{\alpha}}\left(1+\alpha \frac{\phi \bar{\phi}}{2 r}+\ldots\right)+\frac{\Delta(\rho)}{r^{\alpha}} \phi \bar{\phi}
$$

where

$$
\left.X(\rho, 0) \equiv X(\rho) \quad \Delta(\rho) \equiv \partial_{\phi} \partial_{\bar{\phi}} X(\rho, \phi)\right|_{\phi=0}
$$

so that at the minimum $\rho=\rho_{c}$ we find

$$
V\left(\rho_{c}, \phi\right)=V_{0}\left(\rho_{c}\right)+\left(\frac{\alpha V_{0}\left(\rho_{c}\right)}{2 \rho_{c}}+\frac{\Delta\left(\rho_{c}\right)}{\rho_{c}^{\alpha}}\right) \phi \bar{\phi}+\ldots
$$

In principle the second contribution to the mass term might substantially cancel the first, alleviating the problem of the inflaton mass. This would certainly require fine-tuning at the level of one percent (in order to make $\eta$ sufficiently small to allow sixty e-foldings). More importantly, the dependence of $W$ on $\phi$ is not known, so the question of which models admit such fine-tuning cannot be answered at present. We should emphasize that the problem we are discussing is quite general, but one might well be able to find non-generic configurations in which the problem is absent.

Let us discuss these issues more concretely for the case of a brane-antibrane pair transverse to a stabilized Calabi-Yau. In principle one should be able to compute the inflaton potential directly, by substituting the complete superpotential into the supergravity F-term potential

$$
V^{F}=e^{K}\left(g^{i \bar{j}} D_{i} W \overline{D_{j} W}-3|W|^{2}\right)
$$

and possibly including the effects of D-term contributions. This turns out to be a rather subtle problem, essentially because of the breaking of supersymmetry in the braneantibrane system.

We will begin instead by understanding the (supersymmetric) system of a single D3brane transverse to a Calabi-Yau. We will find that superpotential stabilization of the volume necessarily generates mass terms for the scalars $\phi$ which describe the motion of the D3-brane. An implicit assumption in brane-antibrane inflation scenarios is that the brane 
and antibrane are free, in the absence of interactions, to move around the Calabi-Yau; the gentle force from their Coulomb interaction is then expected to lead to a relatively flat inflaton potential. Significant mass terms for the D3-brane (or any external forces on the D3-brane) invalidate this assumption and make inflation impossible.

Let us therefore consider the effective potential governing a D3-brane transverse to a Calabi-Yau manifold. We substitute the superpotential $W(\rho)$ and the Kähler potential (5.1) into (5.11), where the physical volume modulus $r$ is given by (5.3). The resulting four-dimensional effective potential is

$$
V^{F}=\frac{1}{6 r}\left(\partial_{\rho} W \overline{\partial_{\rho} W}\left(1+\frac{1}{2 r} \frac{k,_{\phi} k, \bar{\phi}}{k,_{\phi \bar{\phi}}}\right)-\frac{3}{2 r}\left(\bar{W} \partial_{\rho} W+W \overline{\partial_{\rho} W}\right)\right) .
$$

In the vicinity of a point in moduli space where $k(\phi, \bar{\phi})=\phi \bar{\phi}$, this can be simplified to

$$
V^{F}=\frac{1}{6 r}\left(\left|\partial_{\rho} W\right|^{2}-\frac{3}{2 r}\left(\bar{W} \partial_{\rho} W+W \overline{\partial_{\rho} W}\right)\right)+\left(\frac{\left|\partial_{\rho} W\right|^{2}}{12 r^{2}}\right) \phi \bar{\phi}
$$

We must now incorporate the effects of an anti-D3-brane. In the scenario of [7] the superpotential (5.8) stabilized the compactification volume and generated a negative cosmological term $V_{0}$. The positive, warped tension of an anti-D3-brane was added to this to produce a small positive cosmological constant. In our notation, the anti-D3-brane induces an additional term in the effective potential (5.12),

$$
V=\frac{1}{6 r}\left(\partial_{\rho} W \overline{\partial_{\rho} W}\left(1+\frac{1}{2 r} \frac{k_{, \phi} k, \bar{\phi}}{k_{, \phi \bar{\phi}}}\right)-\frac{3}{2 r}\left(\bar{W} \partial_{\rho} W+W \overline{\partial_{\rho} W}\right)\right)+\frac{D}{(2 r)^{2}}
$$

where $D$ is a positive constant. Notice that this induced term differs from the one in [7] by a factor of $r$. This arises because the anti-D3 tension in the warped compactifications of [5] scales like $\frac{1}{r^{3}} e^{4 A}$, and in the highly warped regime, $e^{4 A} \sim r \exp \left(-\frac{8 \pi K}{3 g_{s} M}\right)$. This does not alter the conclusions of [7], though it changes the numerology.

Suppose that the potential (5.14) has a de Sitter minimum $V_{d S}$ at $\rho=\rho_{c}, \phi, \bar{\phi}=0$. We will now compute the mass of the D3-brane moduli in an expansion about this minimum. To simplify the analysis we assume that at the minimum $\rho$ is real, and also that for real $\rho$, $W(\rho)$ is real. The canonically normalized scalar which governs the motion of the D3-brane is not $\phi$ but is instead a rescaled field $\varphi=\phi \sqrt{3 /(\rho+\bar{\rho})}$; it is the mass of $\varphi$ which we will compute. 
First, we rewrite (5.14) as

$$
V=\left(W^{\prime}(\rho)^{2} \rho-3 W(\rho) W^{\prime}(\rho)+\frac{D}{4}\right)(\rho-\phi \bar{\phi} / 2)^{-2}
$$

where primes denote derivatives with respect to $\rho$, and define $V_{0}$ by

$$
V_{0}\left(\rho_{c}\right)=\frac{1}{\rho_{c}^{2}}\left(W^{\prime}\left(\rho_{c}\right)^{2} \rho_{c}-3 W\left(\rho_{c}\right) W^{\prime}\left(\rho_{c}\right)+\frac{D}{4}\right) .
$$

Then

$$
V=\frac{V_{0}\left(\rho_{c}\right)}{(1-\varphi \bar{\varphi} / 3)^{2}} \approx V_{0}\left(\rho_{c}\right)\left(1+\frac{2}{3} \varphi \bar{\varphi}\right) .
$$

This means that the field $\varphi$ acquires the mass

$$
m_{\varphi}^{2}=\frac{2}{3} V_{d S}=2 H^{2}
$$

This is in fact precisely the result one would obtain for a conformally coupled scalar in a spacetime with cosmological constant $V_{d S}$. This is most easily understood by setting $D=0$ and studying the resulting $\mathrm{AdS}_{4}$. The four-dimensional AdS curvature is $R_{A d S}=4 V_{0}$, so that (5.18) corresponds to a coupling

$$
\delta V=\left(\frac{1}{6} R_{A d S}\right) \varphi \bar{\varphi} .
$$

If the D3-brane is in a highly warped region this result could have been anticipated, since this highly warped region is dual to an almost conformal four-dimensional field theory [25] and the scalar field describing the motion of the brane is conformally coupled (see [21]) 9 . The derivation of (5.19) is also valid even when the D3-brane is far from the near horizon region.

We now see that the D3-brane moduli masses are necessarily of the same scale as the inflationary energy density $V_{0}$, since during inflation, the extra antibrane(s) simply sit at the end of the throat and provide an energy density well-modeled by (5.14). It is straightforward to verify that such masses lead to a slow-roll parameter $\eta=2 / 3$, incompatible with sustained slow-roll inflation.

It is instructive to compare this result with the well-known $\eta$-problem, which bedevils most models of F-term inflation in $\mathcal{N}=1$ supergravity. One begins by asking whether

9 Note that the kinetic term for $\varphi$ is of the form $\int d^{4} x \nabla \varphi \nabla \bar{\varphi}$. 
slow-roll inflation is possible in a model of a single field $\phi$ with any type of Kähler potential and any superpotential $W(\phi)$. For a minimal Kähler potential and a generic superpotential $W(\phi)$ one typically has a inflaton mass $m_{\phi}^{2}=\mathcal{O}\left(H^{2}\right)$, and hence no inflation, just as in the generic case considered in the present paper. But this does not mean that inflation in $\mathcal{N}=1$ supergravity is impossible. Various superpotentials with non-generic dependence on $\phi$ have been found, some of which permit inflation. For example, in supergravity with the canonical Kähler potential and a linear superpotential for the inflaton, the mass term contribution to the potential cancels:

$$
K=\bar{\phi} \phi, \quad W=\phi \quad \Rightarrow \quad V=e^{\bar{\phi} \phi}\left((1+\bar{\phi} \phi)^{2}-3 \bar{\phi} \phi\right)=1+\frac{1}{2}(\bar{\phi} \phi)^{2}+\cdots
$$

A similar effect occurs for the superpotential $W=\phi\left(\sigma_{1} \sigma_{2}-M^{2}\right)$, which leads to a simple realization of F-term hybrid inflation [26]. Moreover, the dangerous mass terms for the inflaton do not appear at all in D-term inflation [27].

It is quite possible, therefore, that one could find a consistent inflation scenario in string theory by studying superpotentials which depend on the inflaton field. As mentioned above, this would undoubtedly require a fine-tuned configuration in which two contributions to the mass cancel to high precision. We treat this question in detail in Appendix F, where we show that the introduction of a superpotential depending on the inflaton field $\phi$ leads to a modification of the mass-squared $m_{\phi}^{2}$ of the inflaton field which could make it much smaller (or much greater) than $2 H^{2}$. This issue merits further investigation, which should become possible as we learn more about the detailed dependence of $W(\rho, \phi)$ on the background geometry and on the fluxes in string compactifications.

\subsection{Scenario II: Kähler Stabilization}

One model of stabilization that would be compatible with the inflationary scenario of $\S 3,4$ is the following. We have seen that the true Kähler-invariant expansion parameter which controls the $\alpha^{\prime}$ expansion in these models, is $r$. Furthermore, $r$ and $\phi$ have independent kinetic terms.

A method of directly stabilizing $r$ could freeze the volume directly, without stopping inflation. Since $r$ is not a chiral superfield itself, stabilization via effects in the superpotential cannot accomplish this. However, given that $W_{0} \neq 0$, one can imagine that corrections to the Kähler potential could directly stabilize $r$. 
In fact, Kähler stabilization has been proposed earlier for rather different reasons (see e.g. [28], which discusses Kähler stabilization of the heterotic string dilaton). Here we would need the $\alpha^{\prime}$ corrections to (5.1) to break the no-scale structure and fix $r$. Some of these corrections have been calculated (see e.g. [29]). The subset of terms presented in $29]$ does not lead to this kind of stabilization, though there are likely to be other terms at the same orders which could change this conclusion. However, Kähler stabilization would be very difficult to find in a controlled calculation, so one might simply have to state it as a model-building assumption.

If one does assume that $r$ is stabilized by corrections to the Kähler potential, then the models of $\S 3,4$ could be realized in the framework of [5]. In Appendix $\mathrm{C}$ we show that in these models one can easily satisfy observational constraints such as the number of e-foldings and the size of the density perturbations.

\section{Conclusion}

One of the most promising ideas for obtaining inflation in string theory is based on brane cosmology. However, brane-antibrane inflation [11] suffers from various difficulties when one tries to embed it in full string compactifications with moduli stabilization, such as the (metastable) de Sitter vacua of [7].

We have argued here that some of these difficulties can be resolved by introducing highly warped compactifications. The warped brane-antibrane models introduced in general form in $\S 3$ and in a compact string theory example in $\S 4$ give rise to slow-roll inflation with an exponentially flat potential. In the compact example, the slow-roll parameters and the density perturbations can be fixed at suitable values by an appropriate choice of discrete fluxes in the warped region.

The above discussion assumes a suitable stabilization mechanism for the volume modulus of the compactification manifold. As described in $§ 5$, this is a highly nontrivial issue. Indeed, we have found that if one stabilizes the moduli as in [7] then this field acquires an effective mass-squared $m_{\phi}^{2}=\mathcal{O}\left(H^{2}\right)$, making inflation impossible. As discussed in $\S 5.1$, fine-tuned dependence of the superpotential on $\phi$ could reduce this mass. With generic dependence on $\phi$ the problem persists. 
The arguments leading to our conclusion that generic methods of stabilization stop inflation are rather general, and should apply to any system where the energy density depends on the volume modulus as $r^{-\alpha}$ with $\alpha>0$. There are general arguments that this should always be the case, for the sources of energy we know about in string theory [30]. Thus, it appears very difficult to achieve slow-roll brane inflation in a manner compatible with stabilization of the compactified space in string theory. At the very least, it is challenging to find a model which works for generic forms of the stabilizing superpotential, which itself varies in a way that depends on all of the microscopic details of the compactification. In those non-generic cases where inflation is possible, the inflationary predictions will depend on the details of the moduli stabilization.

One should note that the degree of fine-tuning required for slow-roll inflation in these models is not extraordinary (see Appendix F), and may well be attainable within the large class of known models. Moreover, even though fine-tuning is certainly undesirable, it may not be a grave problem. Indeed, if there exist many realizations of string theory, then one might argue that all realizations not leading to inflation can be discarded, because they do not describe a universe in which we could live. Meanwhile, those non-generic realizations which lead to eternal inflation (see Appendix D) describe inflationary universes with an indefinitely large and ever-growing volume of inflationary domains. This makes the issue of fine-tuning less problematic. It will not escape the reader's notice that this argument is anthropic in nature [31, 32, 33]. It is worth pointing out that it is an independent, presumably well-defined mathematical question, whether or not string theory has solutions which are consistent with present experiments (e.g. which contain the standard model of particle physics, have sufficiently small cosmological term, and allow early inflation). This question can of course be studied directly (see e.g. [34] for recent work in this direction), and is an important one for string theorists to answer. Only if string theory does admit such solutions, does anthropic reasoning in this context become tenable. The large diversity of string vacua makes it reasonable to be optimistic on this score.

We have primarily focused on the implications of superpotential stabilization of the moduli for D3-brane/anti-D3-brane inflation. Our analysis has implications for other models of brane inflation as well. These include $D p-\overline{D p}$ systems and $D p$-branes at angles with $p=5,7$. In these cases, Chern-Simons couplings will generically induce a $D 3$-brane charge on the branes due to the presence of a non-trivial $B_{N S}$ field. Such a charge will also be generated due to the curvature couplings for generic topologies of the cycles the 
branes wrap. If the induced charge is of order unity or more, the discussion of the previous section will apply. The volume modulus and the inflaton field will mix non-trivially in the Kähler potential and as a result a superpotential of the kind considered in $\S 5.1$, or in fact any source of energy which scales like $1 / r^{\alpha}$, will generically impart an unacceptably big mass to the inflaton. It would be interesting to explore the special cases where such a charge is not induced, to see if one can make simple working models of brane inflation.

Other existing proposals for brane inflation depend on Fayet-Iliopoulos terms in the low-energy field theory [27]. The status of these FI terms in the effective $\mathcal{N}=1$ supergravity arising from compactified string theory therefore merits careful investigation. String theory models with D-terms were realized in brane constructions [14, 17] without consideration of volume stabilization. A consistent embedding of this model into compactified string theory is under investigation [35].

\section{Acknowledgements}

We would like to thank C. Burgess, M. Dine, M. Fabinger, A. Guth, L. Kofman, S. Prokushkin, F. Quevedo, N. Seiberg, A. Sen, M. M. Sheikh-Jabbari, S. Shenker, E. Silverstein, L. Susskind, S. Thomas, H. Tye and E. Witten for helpful discussions. This research was supported by NSF grant PHY-9870115. The work of S.K. was also supported in part by a David and Lucile Packard Foundation Fellowship for Science and Engineering and the DOE under contract DE-AC03-76SF00515. The research of JM is supported in part by DOE grant DE-FG02-90ER40542. The work of L.M. was supported in part by a National Science Foundation Graduate Research Fellowship. S.P.T. acknowledges support from the Swarnajayanti Fellowship, DST, Govt. of India, and most of all from the people of India.

\section{Appendix A. General Discussion of Brane-Antibrane Potentials}

Here we compute the gravitational force between a D3-brane and an anti-D3-brane which are transverse to a general compact six-dimensional space. We assume that there is no warping before we add the D-branes. Our objective is to compute the expression for the slow-roll parameter $\eta$ (2.4) in this setup. For this purpose we note that the brane tension as well as the ten-dimensional Planck mass drop out from the expression for $\eta$ if 
we express it in terms of the physical distance. We can therefore set $M_{P l, 10}=1, T_{D 3}=1$, to avoid clutter in the equations.

The action for the system has the form

$$
S=\int d^{6} x \frac{1}{2}(\nabla \varphi)^{2}+\sum_{i}\left(1+\gamma \varphi\left(x_{i}\right)\right)
$$

where $\gamma$ is a constant we will determine below. Here $\varphi$ is the gravitational potential on the internal space. The equation of motion from (A.1) is

$$
-\nabla^{2} \varphi+\gamma \sum_{i} \delta\left(x-x_{i}\right)=0
$$

Treating one brane as the source and the other as a probe and comparing with (2.1) we see that $\gamma^{2}=2.10$ The expression for the energy of $N$ branes is thus

$$
V \sim N+\frac{1}{2} \sum_{i} \gamma \varphi_{\text {others }}\left(x_{i}\right)
$$

where the subscript in $\varphi$ indicates that we compute the potential due to the other branes, with $j \neq i$, and evaluate it at $x_{i}$. There is also a self-energy correction. We assume that the latter is independent of position. This is true in homogeneous spaces, such as tori.

The equation of motion (A.2) is not consistent since all the charges on the left hand side of (A.2) have the same sign. A minimal modification that makes the equation consistent is to write it as

$$
-\nabla^{2} \varphi+\gamma \sum_{i}\left(\delta\left(x-x_{i}\right)-\frac{2}{v_{6}}\right)=0
$$

where $v_{6}$ is the volume of the compact manifold. This term comes naturally from the curvature of the four-dimensional spacetime, which, in the approximation that we neglect the potential, is de Sitter space. This positive curvature gives rise to a negative contribution to the effective potential in the six internal dimensions. It is reasonable to assume that the negative term is smeared over the compact space as in this minimal modification, as long as the transverse space is approximately homogeneous. 11

10 Note that (2.1) contains a contribution both from gravity and from the Ramond fields, so the gravity contribution is half of that in (2.1).

11 In compactifications with orientifold planes, there would also be localized negative terms. However, these would be cancelled by the tensions of the branes which are present even after brane/antibrane annihilation. The extra energy of the inflationary brane/antibrane pair can be expected to induce a smeared negative contribution over and above the orientifold plane contribution. 
Note that this term does not arise for the Ramond fields since the total charge is zero.

Let us now consider, for simplicity, the case of a single brane and a single antibrane. In order to compute $\eta$ we compute the Laplacian of the potential $V$ with respect to $x_{1}$. We get

$$
\nabla_{x_{1}}^{2} \varphi_{x_{2}}\left(x_{1}\right)=-\frac{2}{v_{6}} \gamma
$$

The subscript in $\varphi$ indicates that this is the potential due to the brane at $x_{2}$. For a pair of branes the potential is $V=2+\gamma \varphi_{x_{2}}\left(x_{1}\right)$. The Laplacian has a constant negative value (A.5). We see that this implies that there exists at least one direction in which the second derivative has a value $V^{\prime \prime} \sim \gamma \varphi^{\prime \prime} \leq-\frac{\gamma^{2}}{3} \frac{1}{v_{6}}$, since there are six transverse dimensions. When we compute the contribution to $\eta$ the factor $v_{6}$ cancels out.

When there are many fields, one should consider $\eta$ as a matrix. In order to have slow roll inflation we need to demand that the matrix has no negative eigenvalue that is too large. If we have a large negative eigenvalue, then even if the scalar field is not initially rolling in that direction, it will typically start moving in this direction after a few e-foldings. The discussion above implies that $\eta$, viewed as a matrix, has an eigenvalue more negative than

$$
\left.\eta\right|_{\text {eigenvalue }} \leq-\frac{2}{3}
$$

This implies that at least one of the moduli acquires a tachyonic mass $m^{2} \leq-2 H^{2}$, which typically prevents a prolonged stage of inflation.

A similar analysis can be carried out for the general case of a Dp-brane/anti-Dp-brane system. It is easy to see that the only change is that the coefficient $\frac{2}{3}$ in (A.6) is replaced by $\frac{4}{(9-p)}$. More interestingly, the above analysis can also be applied to the case of Dp-branes at angles. By this we mean a system of slightly misaligned branes and orientifold planes, [15]. The supersymmetry breaking scale in such a system is controlled parametrically by an angle which measures the relative orientation of the branes. For small values of this angle, the vacuum energy, $V \sim \sum_{i} T_{i}$, obtained by summing over all the branes and planes, can be much smaller than the tension of any individual brane or plane. The force on a brane in such a system arises due to graviton-dilaton and RR exchange. In these systems there can be a cancellation between the graviton-dilaton and the RR force in such a way that the resulting force, computed with non-compact "internal" dimensions, is parametrically smaller than the value of the cosmological constant. Once the internal dimensions are compact, we have to make some modification of the gravitational equation 
in order to make it consistent. The simplest modification is to add a constant term on the right hand side of the corresponding Laplace equation. In this case the constant term will be proportional to the effective four dimensional cosmological constant. Then, repeating the analysis above, one finds that the resulting potential satisfies the inequality

$$
V^{\prime \prime} \leq-\frac{\gamma^{2}}{(9-p)} \frac{1}{v_{6}} T_{p} \sum_{i} T_{i}
$$

As a result, once again one obtains a value of $\eta$, (A.6), with the coefficient $\frac{2}{3}$ replaced by $\frac{4}{(9-p)}$. In other words, both the potential and its second derivative scale in the same way with the small angle which supresses supersymmetry breaking, making $\eta$ independent of this angle.

\section{Appendix B. Computation of the $D 3 / \overline{D 3}$ Potential in Warped Geometries}

To calculate the potential it is actually easier to turn things around and view the D3-brane as perturbing the background and then calculate the resulting energy of the anti-D3-brane in this perturbed geometry. This of course gives the same answer for the potential energy of the brane-antibrane pair.

The coupling of the metric and the five-form to the D3-brane is given by (3.6). On general grounds one expects that the changes in the metric and $F_{5}$ caused by the D3-brane will vary in the directions transverse to the brane. These directions are spanned by the radial coordinate $r$ and the directions along $\mathrm{X}_{5}$. It is useful to observe that the background can be written as follows:

$$
\begin{gathered}
d s^{2}=h^{-\frac{1}{2}}\left(-d t^{2}+d \vec{x}^{2}\right)+h^{\frac{1}{2}}\left(d r^{2}+\frac{r^{2}}{R^{2}} \tilde{g}_{a b} d y^{a} d y^{b}\right) \\
\left(F_{5}\right)_{r t x^{1} x^{2} x 3}=\partial_{r} h^{-1},
\end{gathered}
$$

where $\tilde{g}_{a b} d y^{a} d y^{b}$ is the line element on $\mathrm{X}_{5}$, and $h(r)$ is given by

$$
h(r)=\frac{R^{4}}{r^{4}} \text {. }
$$

It is easy to check that $h(r)$ is a harmonic function in a six-dimensional space spanned by $r$ and the directions along $\mathrm{X}_{5}$, with metric

$$
d s_{6}^{2}=d r^{2}+\frac{r^{2}}{R^{2}} \tilde{g}_{a b} d y^{a} d y^{b} .
$$


Adding one additional D3-brane at a radial location $r_{1}$ results in a perturbed background which is of the form (B.1), but with a harmonic function now given by

$$
h(r)=\frac{R^{4}}{r^{4}}+\delta h(r) .
$$

$\delta h$ solves the equation $\nabla_{6}^{2} \delta h(r)=C \delta^{6}\left(\vec{r}-\overrightarrow{r_{1}}\right)$ in the six-dimensional space (B.4).12 For $r \ll r_{1}$ a simple calculation shows that

$$
\delta h(r)=\frac{R^{4}}{N} \frac{1}{r_{1}^{4}}
$$

independent of $r$ and the detailed metric on $\mathrm{X}_{5}$. In (B.6) the coefficient $N$ arises because the ambient background is supported by $N$ units of charge, whereas the perturbation we are interested in arises due to a single D3-brane. From (B.5) the resulting harmonic function is

$$
h(r)=R^{4}\left(\frac{1}{r^{4}}+\frac{1}{N} \frac{1}{r_{1}^{4}}\right) .
$$

To determine the potential we now couple this new background to the anti-D3-brane. The anti-D3-brane is described by an action of the form (3.6), except that, as was mentioned before, the sign of the Chern-Simons term is reversed relative to the case of a D3-brane. We also remind the reader that the antibrane is located at $r=r_{0}$; we will assume that $r_{1} \gg r_{0}$. Combining all these results, after a simple calculation one recovers the desired potential (3.9).

This calculation of the potential is valid for one brane-antibrane pair. For one brane and $p$ antibranes, to leading order, (3.9) is simply multiplied by $p$. Corrections to this leading-order potential are suppressed for small $p$.

\section{Appendix C. Warped Inflation}

In this appendix we discuss how inflation would look if one managed to fix the overall volume modulus without giving a mass to the brane motion. We argued above that the low energy dynamics of the system is described by the action (3.10). The radial position of the

12 The constant $C$ is determined by the tension of the D3-brane. 
D3-brane, $r_{1}$, will play the role of the inflaton below. We define a canonically normalized field

$$
\phi=\sqrt{T_{3}} r_{1}
$$

and $\phi_{0}=\sqrt{T_{3}} r_{0}$. The effective action is then given by

$$
S=\int d^{4} x \sqrt{-g}\left(\frac{\mathcal{R}}{16 \pi G_{N}}+\frac{1}{2} g^{\mu \nu} \partial_{\mu} \phi \partial_{\nu} \phi-\frac{4 \pi^{2} \phi_{0}^{4}}{N}\left(1-\frac{1}{N} \frac{\phi_{0}^{4}}{\phi^{4}}\right)\right)
$$

We have assumed that there are no significant additional terms in the effective action (C.2).

This inflaton potential is extremely flat: the first term in the potential, which is independent of the inflaton, is larger than the second term by a factor proportional to $\left(\frac{r_{1}}{r_{0}}\right)^{4}$. This factor can be interpreted as the relative redshift between the brane location $r_{1}$ and the antibrane location $r_{0}$; as we explained in $\S 4$, this redshift is exponentially sensitive to the parameters of the model:

$$
r_{0} / R=e^{-\frac{2 \pi K}{3 g_{s} M}}
$$

where $g_{s}$ is the string coupling and $K, M$ are integers that specify fluxes turned on in the compactification.

The slow-roll parameters can now be calculated in standard fashion. We will use conventions where $8 \pi G_{N}=M_{P l}^{2}$. One finds that

$$
\begin{gathered}
\epsilon \equiv \frac{M_{P l}^{2}}{2}\left(\frac{V^{\prime}}{V}\right)^{2} \simeq \frac{8}{N^{2}} M_{P l}^{2} \frac{\phi_{0}^{8}}{\phi^{10}} \\
\eta \equiv M_{P l}^{2} \frac{V^{\prime \prime}}{V} \simeq-\frac{20}{N} M_{P l}^{2} \frac{\phi_{0}^{4}}{\phi^{6}} .
\end{gathered}
$$

Slow-roll requires that $|\eta| \ll 1,|\epsilon| \ll 1$. Of these the condition on $\eta$ is more restrictive. It can be met by taking

$$
\phi \gg\left(\frac{20}{N} M_{P l}^{2} \phi_{0}^{4}\right)^{1 / 6}
$$

The number of e-foldings is given by

$$
N_{e}=\frac{1}{M_{P l}^{2}} \int \frac{V}{V^{\prime}} d \phi \simeq \frac{N}{24} \frac{1}{M_{P l}^{2}} \frac{\phi^{6}}{\phi_{0}^{4}} .
$$

Requiring $N_{e} \sim 60$ can be achieved by taking $\phi$ to be sufficiently large and is compatible with the bound (C.5). 
Finally, the adiabatic density perturbations are given by ([2], page 186)

$$
\delta_{H}=\frac{1}{\sqrt{75} \pi} \frac{1}{M_{P l}^{3}} \frac{V^{3 / 2}}{V^{\prime}}=\frac{\sqrt{N_{e}}}{2 \sqrt{75}} \frac{\phi^{5}}{\phi_{0}^{2} M_{P l}^{3}} .
$$

This quantity should be equal to $1.9 \cdot 10^{-5}$ at $N_{e} \sim 60$, when the perturbations responsible for the large scale structure of the observable part of the universe are produced.

After some algebra, $\delta_{H}$ can be expressed in terms of $N_{e}$ as follows:

$$
\delta_{H}=C_{1} N_{e}^{5 / 6}\left(\frac{T_{3}}{M_{P l}^{4}}\right)^{1 / 3}\left(\frac{r_{0}}{R}\right)^{4 / 3} .
$$

$C_{1}$ is a constant which is somewhat model dependent; using (C.7) and (C.2), one has

$$
C_{1}=\frac{3^{1 / 3} 2^{3 / 2}}{5 \pi}\left(\frac{N}{T_{3} R^{4}}\right)^{1 / 6}
$$

and after using (4.5), (3.7) one finds that $C_{1}=0.39$ for the model of $\S 4.13$

The four-dimensional Planck scale $\left(M_{P l}^{-2} \equiv 8 \pi G_{N}\right)$ is given by

$$
M_{P l}^{2}=\frac{2 V_{6}}{(2 \pi)^{7} \alpha^{\prime 4} g_{s}^{2}}
$$

where $V_{6}$ is the volume of the Calabi-Yau. This formula is strictly applicable only to a Kaluza-Klein compactification, not a warped compactification of the kind considered here. However, the approximation is a good one since the graviton zero mode has most of its support away from the regions with large warping (where its wave function is exponentially damped.) We may express the brane tension as

$$
\frac{T_{3}}{M_{P l}^{4}}=\frac{(2 \pi)^{11}}{4} g_{s}^{3} \frac{\alpha^{\prime 6}}{V_{6}^{2}}
$$

This dimensionless ratio evidently depends on the string coupling constant and the volume of the six compact dimensions. The value $T_{3} / M_{P l}^{4} \sim 10^{-3}$ is quite reasonable: it corresponds to $g_{s} \sim 0.1$ and a Calabi-Yau volume of a characteristic size $\left(V_{6}\right)^{1 / 6} \sim 5 \sqrt{\alpha^{\prime}}$. Larger values of $V_{6}$ lead to smaller values for $T_{3} / M_{P l}^{4}$, which make it easier to meet the density perturbation constraints.

$13 C_{1}$ increases by a factor $\sqrt{p}$ when there are $p$ antibranes. While making numerical estimates we set $p=2$. 
More important, for present purposes, is the factor $\left(r_{0} / R\right)^{4 / 3}$, which has its origins in the redshift suppression of the potential that was emphasized in the discussion above. By taking this factor to be small enough we see that the constraint on $\delta_{H}$, (C.7), can be met. As an example, taking $T_{3} / M_{P l}^{4} \sim 10^{-3}$ and $N_{e}=60$, we find that $\delta_{H} \approx 1.91 \cdot 10^{-5}$ for $r_{0} / R=2.5 \cdot 10^{-4}$. This condition on $r_{0} / R$ can easily be met for reasonable values of the flux integers $K, M$. Taking $g_{s}=0.1$, we get $r_{0} / R=2.5 \cdot 10^{-4}$, with $K / M \simeq 0.4$. The latter condition can be achieved using moderate values of flux, e.g. $K=8, M=20$.

Now that we have ensured that the various constraints can be met in our model, it is worth exploring the resulting inflationary scenario a little more. The energy scale during inflation can be expressed in terms of $\delta_{H}$. One finds from (C.8), and using the fact that the potential is well approximated by the first term in (3.9), that

$$
\frac{V}{M_{P l}^{4}}=\frac{2 \delta_{H}^{3}}{C_{1}^{3} N_{e}^{5 / 2}} .
$$

Taking $\delta_{H}=1.91 \cdot 10^{-5}, N_{e}=60, C_{1}=0.39$ and $M_{P l}=2.4 \cdot 10^{18} \mathrm{GeV}$ one finds that the energy scale is

$$
\Lambda \equiv V^{1 / 4}=1.3 \cdot 10^{14} \mathrm{GeV} .
$$

This is considerably lower than the GUT scale $\sim 10^{16} \mathrm{GeV}$. This low scale of inflation is a generic feature of the scenario.

Next, it is straightforward to see that $\delta_{H}$ is given in terms of $V$ and $\epsilon$ by

$$
\delta_{H}=\frac{1}{5 \pi \sqrt{6 \epsilon}}\left(\frac{V}{M_{P l}^{4}}\right)^{1 / 2} .
$$

Solving for $V$ from (C.12) gives

$$
\epsilon=\frac{\delta_{H}}{75 \pi^{2} C_{1}^{3} N_{e}^{5 / 2}} .
$$

Taking $\delta_{H}=1.91 \cdot 10^{-5}, C_{1}=0.39, N_{e}=60$ gives

$$
\epsilon=1.54 \cdot 10^{-11},
$$

a very small number. The ratio of the anisotropy in the microwave background generated by gravitational waves to that generated by adiabatic density perturbations is given (at large $l$ ) by

$$
r \simeq 12.4 \epsilon
$$


In our model this is very small, so the anisotropy is almost entirely due to density perturbations.

Finally, $\eta$ can be related to $N_{e}$, and is given by

$$
\eta=-\frac{5}{6} \frac{1}{N_{e}}
$$

Setting $N_{e}=60$ gives

$$
\eta=-0.014
$$

Clearly, as we mentioned above, $|\eta| \gg \epsilon$. The tilt parameter is given by

$$
n=1-6 \epsilon+2 \eta \simeq 1+2 \eta \approx 0.97
$$

in excellent agreement with observational data from WMAP.

In summary, in our model the scale of inflation $\Lambda$ (C.13) is generically low. Most of the anisotropy originates from adiabatic density perturbations, since $\epsilon$ is extremely small, and the tilt in the spectrum, (C.20), is determined by $\eta$. The values for these parameters are nearly model-independent: they are almost entirely determined by the observed value for $\delta_{H}$ and by the number of e-foldings, $N_{e}$.

\section{Appendix D. Eternal Inflation}

At large $\phi$, the potential $V(\phi)$ in (C.2) becomes extremely flat. For flat potentials, the force pushing the field $\phi$ down becomes very small, whereas the amplitude of inflationary fluctuations remains practically constant. As a result, the motion of the field $\phi$ at large $\phi$ is mainly governed by quantum jumps. This effect is known to lead to eternal inflation [36,37.

Eternal inflation leads to formation of a fractal structure of the universe on a very large scale. It occurs for those values of the field $\phi$ for which the post-inflationary amplitude of perturbations of the metric $\delta_{H}$ would exceed unity [1]. In our case $\delta_{H}$ is proportional to $\phi^{5}$, cf. (C.7). Since the amplitude of the density perturbations is $\delta_{H} \sim 1.9 \cdot 10^{-5}$ in the observable part of the universe, eternal inflation should occur for all values of the field $\phi$ that are greater than $10 \cdot \phi_{60}$. Here $\phi_{60}$ is the value of the field at the moment starting from which the universe inflated $e^{N_{e}} \sim e^{60}$ times. In other words, if $r_{60}$ is the brane 
separation corresponding to the moment when the large-scale structure of the observable part of the universe was produced, then the regime of eternal inflation occurred when the brane separation was ten times greater than $r_{60}$. The possibility of eternal inflation in our model is very interesting since this regime makes the existence of inflation much more plausible: even if the probability of initial conditions for eternal inflation is small, the universes (or the parts of the universe) where these conditions are satisfied rapidly acquire indefinitely large (and ever growing) volume [38].

\section{Appendix E. Exit from Inflation}

In this appendix we comment on the exit from inflation through brane-antibrane annihilation.

The brane-antibrane potential used in our analysis of inflation is no longer valid when the brane separation is comparable to the string length. At that stage a tachyon appears and then condenses. (In this sense, our model, like all the brane inflation models described in [4], is a particular version of the hybrid inflation scenario [39].) One may attempt to use the properties of this brane-antibrane tachyon [40,41] to describe the exit from inflation. Here we will show that one of the possible problems of this scenario, the overproduction of cosmic strings 42,43, is ameliorated by the warped geometry.

In the case of a merging brane-antibrane pair, the tachyon is a complex field and there is a $U(1)$ symmetry. Formation of cosmic strings associated with the $U(1)$ symmetry breaking leads to large-scale perturbations of the metric which are compatible with the current observations of the cosmic microwave anisotropy [44] only if $G_{N} T_{1}=\frac{T_{1}}{8 \pi M_{P l}^{2}} \lesssim$ $10^{-7}$, where $T_{1}$ is the cosmic string tension [45]. This tension can be evaluated either by the methods of [46], or by identifying cosmic strings with D1-branes. In the usual case (i.e. ignoring warping) one has

$$
T_{1}=\frac{1}{2 \pi g_{s} \alpha^{\prime}}
$$

The requirement $G_{N} T_{1}=\frac{T_{1}}{8 \pi M_{P l}^{2}} \lesssim 10^{-7}$ reads

$$
G_{N} T_{1}=\frac{g_{s}}{16 \pi} \frac{\left(2 \pi l_{s}\right)^{6}}{V_{6}} \lesssim 10^{-7}
$$


i.e.

$$
V_{6} \gtrsim 2 \times 10^{5} g_{s}\left(2 \pi l_{s}\right)^{6}
$$

This shows that the cosmic string contribution to the perturbations of the metric produced after inflation is unacceptably large unless the volume of the compactified space $V_{6}$ is at least five orders of magnitude greater than $g_{s}\left(2 \pi l_{s}\right)^{6}$.

In the brane inflation models of $\S 3, \S 4$, however, the relevant tension is redshifted by the warped geometry, which leads to exponential suppression of $T_{1}$ :

$$
T_{1}=\frac{1}{2 \pi g_{s} \alpha^{\prime}} e^{-\frac{4 \pi K}{3 g_{s} M}}
$$

As a result, the undesirable contribution of cosmic strings (D1-branes) to perturbations of the metric becomes exponentially suppressed.

\section{Appendix F. Fine-tuning of the Potential when the Superpotential Depends on the Inflaton Field}

In this appendix we study a toy model in order to make more precise our statements concerning the degree of fine-tuning which is required for slow-roll brane inflation. We should note here that we will be discussing the degree to which the inflaton potential itself must be tuned. In a given string model, one cannot directly tune the potential, but only vary choices of the background data like fluxes, compactification manifold, or brane positions. It could be that the tuning required in terms of this data is more or less severe than our estimate below, but explicit string calculations of the relevant superpotentials will be necessary to determine this.

Before studying the example, let us mention how small the inflaton mass term must be for a given model of slow-roll inflation to be compatible with experiment. The goal is to have a long stage of inflation producing metric fluctuations with a fairly flat spectrum. Recent observations suggest that, modulo some uncertainties, the tilt is $n_{s} \approx 1+\frac{2 m_{\phi}^{2}}{3 H^{2}}=$ $0.97 \pm 0.03$ 44,47]. This is compatible with an inflaton mass $\left|m_{\phi}^{2}\right| / H^{2} \sim 10^{-1}-10^{-2}$.

This could be achieved through fine-tuning of $m_{\phi}^{2}$ by only about one part in 100 . Thus, the fine-tuning that we need to perform is not extraordinary. Given the large number of 
possible compactifications, the existence of some configurations which allow inflation seems quite likely.

We now turn to an example which illustrates this point. Consider a D3-brane transverse to a warped compactification; we would like to know how the (brane-antibrane) inflaton mass terms vary as the inflaton-dependence of the superpotential varies.

The Kähler potential for the volume modulus and the D3-brane field $\phi$ takes the form $K(\rho, \bar{\rho}, \phi, \bar{\phi})=-3 \log (\rho+\bar{\rho}-k(\phi, \bar{\phi}))$. We will work in the vicinity of the point $\phi=\bar{\phi}=0$ in moduli space, where $k(\phi, \bar{\phi})=\phi \bar{\phi}$. We choose a superpotential of the form

$$
W(\rho, \phi)=W_{0}+g(\rho) f(\phi)
$$

where $g(\rho)$ is an arbitrary function of $\rho, f(\phi)=\left(1+\delta \phi^{2}\right)$, and $W_{0}$ and $\delta$ are constants. This is a slight generalization of the superpotential in [7], which corresponds to $\delta=0$ and $g(\rho)=A e^{-a \rho}$.

One can now calculate the supergravity potential $V^{F}=e^{K}\left(g^{i \bar{j}} D_{i} W \overline{D_{j} W}-3|W|^{2}\right)$ for the two complex fields $\rho, \phi$. The exact potential has a simple dependence on $\operatorname{Im} \rho$ and $\operatorname{Im} \phi$ which shows that the point $\operatorname{Im} \rho=\operatorname{Im} \phi=0$ is an extremum of the potential (it is a minimum, at least for small $\phi$ ). Therefore we will present here the exact potential $V^{F}(\sigma, \psi)$ as a function of $\operatorname{Re} \rho=\sigma$ and $\operatorname{Re} \phi=\psi$ at $\operatorname{Im} \rho=\operatorname{Im} \phi=0$.

$$
V^{F}(\sigma, \psi)=\frac{1}{6\left(\sigma-\psi^{2} / 2\right)^{2}}\left(2 \delta^{2} \psi^{2} g(\sigma)^{2}+f(\psi) g^{\prime}(\sigma) g(\sigma)\left(-\frac{3 W_{0}}{g(\sigma)}+\sigma f(\psi)-f(\psi)-2\right)\right)
$$

We are interested in the total potential $V^{F}(\sigma, \psi)+V_{\bar{D} 3}$ at small $\psi$, where $V_{\bar{D} 3}$ is the potential due to the antibrane (cf. (5.14)). We may therefore use the stabilization of the volume in the first approximation at $\psi^{2}=0$ and calculate the potential at the AdS critical point $\sigma_{c}=r_{c}$, where, using $\left.D_{\rho} W\right|_{\phi=0}=0$, one finds

$$
W_{0}=-g\left(\sigma_{c}\right)+\frac{2}{3} \sigma_{c} g^{\prime}\left(\sigma_{c}\right), \quad V_{A d S}=-\frac{\left(g^{\prime}\left(\sigma_{c}\right)\right)^{2}}{6 \sigma_{c}}
$$

We now change variables to $\psi^{2}=\frac{2}{3} \sigma_{c} \varphi^{2}$, where $\varphi$ is a field with the canonical kinetic term $(\partial \varphi)^{2}$. We find

$$
V^{F}\left(\sigma_{c}, \varphi\right)=\frac{1}{6 \sigma_{c}\left(1-\varphi^{2} / 3\right)^{2}}\left(-\left(g^{\prime}\right)^{2}+\frac{4 \delta^{2} g^{2}}{3} \varphi^{2}-\frac{2 \delta g g^{\prime}}{3} \varphi^{2}\left(1+\frac{2 \delta}{3} \sigma_{c} \varphi^{2}\right)+\frac{4}{9} \delta^{2} \varphi^{4} \sigma_{c}^{2}\right)
$$


From the antibrane we get the additional contribution mentioned above. Keeping terms up to those quadratic in $\phi$, we finally arrive at

$$
V^{F}\left(\sigma_{c}, \varphi\right)+V_{\bar{D} 3}\left(\sigma_{c}, \varphi\right) \approx V_{d S}+\frac{2 \varphi^{2}}{3}\left(V_{d S}+\frac{1}{6 \sigma_{c}}\left(2 \delta^{2} g^{2}-\delta g^{\prime} g\right)\right)
$$

Here $V_{d S}$ is the value of the potential at the de Sitter minimum,

$$
V_{d S}=V_{A d S}+\frac{D}{4 \sigma_{c}^{2}}=-\frac{\left(g^{\prime}\left(\sigma_{c}\right)\right)^{2}}{6 \sigma_{c}}+\frac{D}{4 \sigma_{c}^{2}} \equiv 3 H^{2}
$$

The mass-squared of the field $\phi$ is

$$
m_{\phi}^{2}=2 H^{2}+\frac{2\left|V_{A d S}\right|}{3}\left[2\left(\delta \frac{g}{g^{\prime}}\right)^{2}-\delta \frac{g}{g^{\prime}}\right]
$$

To make $m_{\phi}^{2}$ small, we need $\delta \frac{g}{g^{\prime}}>0$ as well as $\frac{\left|V_{A d S}\right|}{3}\left[2\left(\delta \frac{g}{g^{\prime}}\right)^{2}-\delta \frac{g}{g^{\prime}}\right] \approx-H^{2}$. If the parameters of the model were arbitrary then this would certainly be possible.

We will express our results in terms of a parameter $\beta=\delta \frac{g}{g^{\prime}}$ :

$$
m_{\phi}^{2}=2 H^{2}-\frac{2}{3}\left|V_{A d S}\right|\left(\beta-2 \beta^{2}\right)=2 H^{2}\left(1-\frac{\left|V_{A d S}\right|}{V_{d S}}\left(\beta-2 \beta^{2}\right)\right)
$$

For $\beta=0$ we recover the "conformal" result

$$
m_{\phi}^{2}=2 H^{2}
$$

As a simple example, if $g(\rho)=A e^{-a \rho}$, as in [7], we find $\beta=-\frac{\delta}{a}$. However, let us assume, as in [7], that $\left|V_{A d S}\right| \gg V_{d S}$. Then for the simple value $\beta=1$ (i.e. $\delta=-a$ ) we have

$$
m_{\phi}^{2}=2 H^{2}\left(1+\frac{\left|V_{A d S}\right|}{V_{d S}}\right) \approx \frac{2}{3}\left|V_{A d S}\right| \gg 2 H^{2}
$$

Thus, whereas it is true that our knowledge of $W(\rho, \phi)$ is not particularly good, our absence of knowledge does not allow us to say much about $m_{\phi}^{2}$. The only thing we can say is that in our particular example, for $\left|V_{A d S}\right| \gg V_{d S}$, this mass can be fine-tuned to take almost any value 14 In particular, one has a flat potential with $m_{\phi}^{2}=0$ for

$$
\beta=\frac{1}{4}\left(1 \pm \sqrt{1-\frac{8 V_{d S}}{\left|V_{A d S}\right|}}\right)
$$

14 Incidentally, Eq. (F.10) implies that if one does not make any fine-tuning, then for the model described in [7], with $V_{d S} \sim 10^{-120}$ in Planck units, the typical mass squared of the D3 brane moduli fields is expected to be $\mathcal{O}\left(\left|V_{A d S}\right|\right)$, which can be extremely large. This result may have interesting phenomenological implications. 
This equation always has solutions for $\left|V_{A d S}\right| \geq 8 V_{d S}$. For $\left|V_{A d S}\right| \gg 8 V_{d S}$, the solutions are:

$$
\beta_{1}=\frac{\delta_{1}}{a}=\frac{1}{2}-\frac{V_{d S}}{\left|V_{A d S}\right|} \approx \frac{1}{2}
$$

and

$$
\beta_{2}=\frac{\delta_{2}}{a}=\frac{V_{d S}}{\left|V_{A d S}\right|} \ll 1
$$

In order to satisfy one of these two conditions and have $m_{\phi}^{2}=0$ one can fine-tune either the ratio $\frac{V_{d S}}{\left|V_{A d S}\right|}$ (as was done in [7]) or the coefficient $\delta$ in the superpotential. In order to prove that inflation in this scenario is impossible, one would need to prove that neither of these types of fine-tuning is possible.

It is instructive to compare this situation with the problem of realizing the chaotic inflation scenario in $\mathcal{N}=1$ supergravity. Let us consider a canonical Kähler potential $K=$ $\bar{\phi} \phi+\bar{\sigma}_{i} \sigma_{i}$, where $\phi$ is the inflaton field and $\sigma_{i}$ are some other fields. If the superpotential is a function of the fields $\sigma_{i}$ but not of the field $\phi$, then the potential of the scalar fields has the general structure as a function of the real part of the field $\phi, V=e^{\phi^{2}} V\left(\sigma_{i}\right)$, which implies that $m_{\phi}^{2}=3 H^{2}$, i.e. $\eta=1$.

One can resolve this problem by introducing a superpotential depending on the inflaton field, just as we did in this appendix. However, in the simplest version of chaotic inflation one needs the inflaton field to be at $\phi \gg 1$, in Planck mass units, and to change significantly, by $\Delta \phi=\mathcal{O}(1)$, during the last 60 e-folds. It is this last part that causes substantial difficulties for inflation in $\mathcal{N}=1$ supergravity. It is always possible to find a superpotential which depends on the inflaton field $\phi$ in such a way that the potential becomes flat in the vicinity of one particular point. However, one must do this for all $\phi$ in a large interval $\Delta \phi=\mathcal{O}(1)$. One needs enormous functional fine-tuning in a large interval at $\phi \gg 1$, where the term $\sim e^{\phi^{2}}$ grows very fast.

Meanwhile, in our case the situation is much better. Instead of a functional fine-tuning in a large interval of $\phi$ we need to make a fine-tuning at a single point $\sigma=\sigma_{c}, \phi=0$. In order to estimate the required degree of fine-tuning, let us e.g. fix $\beta=1 / 2$ and change the ratio $\left|V_{A d S}\right| / V_{d S}$ in Eq. (F.8) in the interval $0<\left|V_{A d S}\right| / V_{d S}<4$. As one can easily see, in this case the mass squared of the inflaton field changes from $2 H^{2}$ to $-2 H^{2}$. In approximately $1 \%$ of this interval the condition $n_{s} \approx 1+\frac{2 m_{\phi}^{2}}{3 H^{2}}=0.97 \pm 0.03$ is satisfied. On the other hand, if this condition is substantially violated, which happens in the main part 
of this interval, then inflation becomes either too short or impossible, and the universe most probably becomes unsuitable for life.

Finally, if inflation can be eternal (and it can be eternal in the models of $\S 3,4$, see Appendix D), then the parts of the universe where eternal inflation is possible have an indefinitely large and ever-increasing volume. For this reason, regions of the universe where eternal inflation does occur, however improbable that may have been, are in some sense favored. One could therefore argue that the problem of fine-tuning in inflationary cosmology is not as dangerous as one could expect, and sometimes it may not even be particularly relevant. 


\section{References}

[1] A.D. Linde, Particle Physics and Inflationary Cosmology, Harwood, Chur, Switzerland (1990).

[2] A.R. Liddle and D.H. Lyth, Cosmological Inflation and Large-Scale Structure, Cambridge University Press, Cambridge, England (2000).

[3] S. Hellerman, N. Kaloper and L. Susskind, "String Theory and Quintessence," JHEP 0106 (2001) 003, hep-th/0104180;

W. Fischler, A. Kashani-Poor, R. McNees and S. Paban, "The Acceleration of the Universe: A Challenge for String Theory," JHEP 0107 (2001) 003, hep-th/0104181.

[4] F. Quevedo, "Lectures on String/Brane Cosmology," hep-th/0210292.

[5] S. Giddings, S. Kachru and J. Polchinski, "Hierarchies from Fluxes in String Compactifications," Phys. Rev. D66 (2002) 106006, hep-th/0105097.

[6] O. DeWolfe and S. Giddings, "Scales and Hierarchies in Warped Compactifications and Brane Worlds," Phys. Rev. D67 (2003) 066008, hep-th/0208123.

[7] S. Kachru, R. Kallosh, A. Linde and S. P. Trivedi, "de Sitter Vacua in String Theory," hep-th/0301240, to appear in Phys. Rev. D.

[8] A. Frey, M. Lippert and B. Williams, "The Fall of Stringy de Sitter," hep-th/0305018.

[9] C. Escoda, M. Gomez-Reino and F. Quevedo, "Saltatory de Sitter String Vacua," hep-th/0307160.

[10] A. Maloney, E. Silverstein and A. Strominger, "de Sitter Space in Non-Critical String Theory," hep-th/0205316.

[11] G. Dvali and S.H. Tye, "Brane Inflation," Phys. Lett. B450 (1999) 72, hepth/9812483.

[12] S. Alexander, "Inflation from D - Anti-D-Brane Annihilation," Phys. Rev. D65 (2002) 023507, hep-th/0105032;

G. Dvali, Q. Shafi and S. Solganik, "D-brane Inflation," hep-th/0105203;

C.P. Burgess, M. Majumdar, D. Nolte, F. Quevedo, G. Rajesh and R.J. Zhang, "The Inflationary Brane-Antibrane Universe," JHEP 07 (2001) 047, hep-th/0105204;

G. Shiu and S.H. Tye, "Some Aspects of Brane Inflation," Phys. Lett. B516 (2001) 421, hep-th/0106274;

D. Choudhury, D. Ghoshal, D.P. Jatkar, S. Panda, "Hybrid Inflation and BraneAntibrane System," hep-th/0305104. 
[13] R. Brustein, S. De Alwis and E. Novak, "Inflationary Cosmology in the Central Region of String/M-theory Moduli space," Phys. Rev. D68 (2003) 023517, hep-th/0205042; R. Brustein, S. De Alwis and E. Novak, "M-theory Moduli Space and Cosmology," Phys. Rev. D68 (2003) 043507, hep-th/0212344.

[14] C. Herdeiro, S. Hirano and R. Kallosh, "String Theory and Hybrid Inflation/Acceleration," JHEP 0112 (2001) 027, hep-th/0110271.

[15] J. Garcia-Bellido, R. Rabadan and F. Zamora, "Inflationary Scenarios from Branes at Angles," JHEP 0201 (2002) 036, hep-th/0112147.

[16] B. Kyae and Q. Shafi, "Branes and Inflationary Cosmology," Phys. Lett. B526 (2002) 379, hep-ph/0111101.

[17] K. Dasgupta, C. Herdeiro, S. Hirano and R. Kallosh, "D3/D7 Inflationary Model and M-theory," Phys. Rev. D65 (2002) 126002, hep-th/0203019.

[18] N. Jones, H. Stoica and S.H. Tye, "Brane Interaction as the Origin of Inflation," JHEP 0207 (2002) 051, hep-th/0203163.

[19] L. Randall and R. Sundrum, "A Large Mass Hierarchy from a Small Extra Dimension," Phys. Rev. Lett. 83 (1999) 3370, hep-ph/9905221.

[20] I. Klebanov and M.J. Strassler, "Supergravity and a Confining Gauge Theory: Duality Cascades and $\chi$ SB Resolution of Naked Singularities," JHEP 0008 (2000) 052, hepth/0007191.

[21] N. Seiberg and E. Witten, "The D1/D5 System and Singular CFT," JHEP 9904 (1999) 017, hep-th/9903224.

[22] S. Kachru, J. Pearson and H. Verlinde, "Brane/Flux Annihilation and the String Dual of a Nonsupersymmetric Field Theory," JHEP 0206 (2002) 021, hep-th/0112197.

[23] E. Witten, "Dimensional Reduction Of Superstring Models," Phys. Lett. B 155 (1985) 151.

[24] O. Ganor, "A Note on Zeroes of Superpotentials in F-theory," Nucl. Phys. B499 (1997) 55, hep-th/9612077.

[25] J. Maldacena, "The Large $N$ Limit of Superconformal Field Theories and Supergravity," Adv. Th. Math. Phys. 2 (1998) 231, hep-th/9711200.

[26] E. J. Copeland, A. R. Liddle, D. H. Lyth, E. D. Stewart and D. Wands, "False Vacuum Inflation with Einstein Gravity," Phys. Rev. D 49 (1994) 6410, astro-ph/9401011; G. R. Dvali, Q. Shafi and R. Schaefer, "Large Scale Structure and Supersymmetric 
Inflation without Fine Tuning," Phys. Rev. Lett. 73 (1994) 1886, hep-ph/9406319;

A. D. Linde and A. Riotto, "Hybrid Inflation in Supergravity," Phys. Rev. D 56 (1997) 1841, hep-ph/9703209.

[27] P. Binetruy and G. R. Dvali, "D-term inflation," Phys. Lett. B 388 (1996) 241, hep$\mathrm{ph} / 9606342$.

[28] T. Banks and M. Dine, "Coping with Strongly Coupled String Theory," hepth/9406132.

[29] K. Becker, M. Becker, M. Haack and J. Louis, "Supersymmetry Breaking and Alpha-Prime Corrections to Flux Induced Potentials," JHEP 0206 (2002) 060, hepth/0204254.

[30] S. Giddings, "The Fate of Four Dimensions," Phys. Rev. D68 (2003) 026006, hepth/0303031.

[31] R. Bousso and J. Polchinski, "Quantization of Four-form Fluxes and Dynamical Neutralization of the Cosmological Constant," JHEP 0006 (2000) 006, hep-th/0004134.

[32] A. Linde, "Inflation, quantum cosmology and the anthropic principle," hep-th/0211048.

[33] L. Susskind, "The Anthropic Landscape of String Theory," hep-th/0302219.

[34] S. Ashok and M. Douglas, "Counting Flux Vacua," hep-th/0307049;

M. Douglas, "The statistics of string/M theory vacua," hep-th/0303194.

[35] R. Kallosh, work in progress.

[36] A. Vilenkin, "The Birth of Inflationary Universes," Phys. Rev. D 27 (1983) 2848.

[37] A. D. Linde, "Eternally Existing Selfreproducing Chaotic Inflationary Universe," Phys. Lett. B 175 (1986) 395.

[38] A. D. Linde, D. A. Linde and A. Mezhlumian, "From the Big Bang Theory to the Theory of a Stationary Universe," Phys. Rev. D 49 (1994) 1783, gr-qc/9306035.

[39] A. D. Linde, "Axions in Inflationary Cosmology," Phys. Lett. B 259 (1991) 38; A. D. Linde, "Hybrid Inflation," Phys. Rev. D 49 (1994) 748, astro-ph/9307002.

[40] A. Sen, "Tachyon Condensation on the Brane Anti-Brane System," JHEP 9808 (1998) 012, hep-th/9805170.

[41] M.R. Garousi, "Tachyon Couplings on Non-BPS D-branes and Dirac-Born-Infeld Action," Nucl. Phys. B584 (2000) 584, hep-th/0003122;

E.A. Bergshoeff, M. de Roo, T.C. de Wit, E. Eyras and S. Panda, "T-Duality and 
Actions for Non-BPS D-branes," JHEP 0005 (2000) 009, hep-th/0003221;

J. Kluson, "Proposal for Non-BPS D-brane Action," Phys. Rev. D62 (2000) 126003, hep-th/0004106.

[42] L. Kofman and A. Linde, "Problems with Tachyon Inflation," JHEP 0207 (2002) 004, hep-th/0205121.

[43] S. Sarangi and S. H. Tye, "Cosmic String Production Towards the End of Brane Inflation," Phys. Lett. B 536 (2002) 185, hep-th/0204074; N. T. Jones, H. Stoica and S. H. Tye, "The Production, Spectrum and Evolution of Cosmic Strings in Brane Inflation," Phys. Lett. B 563 (2003) 6, hep-th/0303269; L. Pogosian, S. H. Tye, I. Wasserman and M. Wyman, "Observational Constraints on Cosmic String Production During Brane Inflation," Phys. Rev. D 68 (2003) 023506, hep-th/0304188.

[44] C. L. Bennett et al., "First Year Wilkinson Microwave Anisotropy Probe (WMAP) Observations: Preliminary Maps and Basic Results," astro-ph/0302207.

[45] P. P. Avelino and A. R. Liddle, "Cosmological Perturbations and the Reionization Epoch," astro-ph/0305357.

[46] A. Sen, "Dirac-Born-Infeld Action on the Tachyon Kink and Vortex," hep-th/0303057.

[47] C. R. Contaldi, H. Hoekstra and A. Lewis, "Joint CMB and Weak Lensing Analysis: Physically Motivated Constraints on Cosmological Parameters," Phys. Rev. Lett. 90 (2003) 221303, astro-ph/0302435. 